\title{
Sperm DNA Fragmentation, Determined Using the Sperm Chromatin Dispersion (SCD) Test, A Study in Republic of Kosovo Population
}

\author{
Afrim Zeqiraj ${ }^{1}$, Sheqibe Beadini ${ }^{3}$, Nexhbedin Beadini ${ }^{3}$, Hesat Aliu $^{3}$, Zafer Gashi ${ }^{1}$, Shkelzen Elezaj ${ }^{4}$, Sadi Bexheti $^{5}$, \\ Sanije Berisha ${ }^{6} \&$ Agim Shabani $^{2}$ \\ ${ }^{1}$ Department of Biochemistry, FAMA Colleague, Republic of Kosovo \\ ${ }^{2}$ Department of Chemistry, University of Tetovo, Republic of Macedonia \\ ${ }^{3}$ Department of Biology, University of Tetovo, Republic of Macedonia \\ ${ }^{4}$ Clinic of Urology, Regional Hospital of Peja, Republic of Kosovo \\ ${ }^{5}$ Faculty of Medicine, University of Tetovo, Republic of Macedonia \\ ${ }^{6}$ Department of Biochemistry, University clinical center, Tetovo, Republic of Macedonia \\ Correspondence: Agim Shabani, Department of Chemistry, University of Tetovo, Republic of Macedonia. Tel: \\ 389-4435-6500. E-mail: agim.shabani@unite.edu.mk
}

\author{
Received: April 9, 2018 \\ Accepted: April 16, 2018 \\ Online Published: April 20, 2018 \\ doi:10.5539/ijb.v10n3p14 \\ URL: https://doi.org/10.5539/ijb.v10n3p14
}

\begin{abstract}
Infertility is a common condition affecting one in six couples of childbearing age. In approximately $40 \%$ of these cases, a male factor is involved. Sperm DNA integrity is essential for accurate transmission of genetic information. Materials and Methods: In this study 152 patients, 64 patients it is infertility group and 88 patients are fertile males. The ejaculate samples were taken in accordance with the patient to whom the reason for the analysis of the ejaculate sample was previously explained. All patients have been in the Dukagjini Region in the Republic of Kosovo. The samples were collected from 2016/18. Sperm Chromatin Dispersion (SCD) test, analysis in the ejaculate was performed at the Biolab Zafi, Laboratory in Peja, in the Republic of Kosovo. Statistical analysis: Data are reported as mean $\pm \mathrm{SD}$. The comparisons between groups were tested by student's t-test, ANOVA. A p-value less than $0.05 \%$ was considered statistically significant. Results: From our research studies, we have achieved significant $(p<.00001)$ scores among the working group and control group across all sperm parameters, and DNA fragmentation. Conclusion: In summary, we have demonstrated that there was a negative correlation between DNA fragmentation, sperm motility, and morphology in infertile males. We conclude that sperm DNA fragmentation appears to be a useful technique to predict outcome in couples undergoing IVF/ICSI. To evaluate whether DFI $23.94 \pm 4.68 \%$ can be used to determine male infertility in our country by Sperm Chromatin Dispersion (SCD), it is necessary to carry out further large-scale research by other authors.
\end{abstract}

Keywords: DNA-fragmentation, Sperm Chromatin Dispersion (SCD), IVF/ICSI

\section{Introduction}

Infertility is a common condition affecting one in six couples of childbearing age. In approximately $40 \%$ of these cases, a male factor is involved. The common definition for male infertility is the presence of an alteration in concentration, motility and/or morphology in at least one sample of two sperm analyses, according to World Health Organization guidelines (WHO, 1999). However, in the last decade there have been rising concerns about factors undermining male fertility potentials which may lie outside the realm of WHO standards. DNA damage in spermatozoa, and subsequent effects of such damage on male reproductive ability, is one of these factors which has been studied in the past few years Because half of the offspring's DNA is originating from paternal unit, it is of utmost importance to consider the detrimental effects of sperm chromatin and DNA damage on reproduction (Marchettini et al., 2007). Semen analysis is a routine test to evaluate sperm quality. Despite some pitfalls, the test result is generally acceptable and considered reliable in the assessment of male fecundity (Høst et al., 2001). Nevertheless, semen analysis is subjective and sperm parameters vary based on patient conditions. However, they do not always reveal the quality and health of sperm (Agarwal et al., 2003). Sperm DNA integrity is essential for accurate transmission of genetic information (Gandini, 2004). Apoptosis, reactive oxygen species (ROS) and abnormalities in chromatin packaging could be major major sources of sperm DNA damage (Kim et al., 2001; 
Saleh, 2003; Manicardi et al., 1998). It seems that genetic abnormalities in the paternal genome are one of the main causes for early pregnancy loss (EPL) ( $\mathrm{Li}, 1998)$. The DNA fragmentation index (DFI) can be used as an independent predictor of fertility in couples undergoing ART (Bungum, 2007). Recently, an easy and fast diagnostic test based on sperm chromatin dispersion (SCD), has been developed (Fernández et al., 2005). Normal sperm without massive DNA fragmentation produce nucleotides with large halos of spreading DNA loops (Yilmaz et al., 2010). It is postulated that if the sperm DNA fragmentation (DF) value exceeds $30 \%$, sperm quality decreases significantly (Virro, 2004). Based on a DF quantitative value, it may be possible to choose the appropriate technique in infertility clinics. On the other hand, using the DF value for all patients is not cost-effective; therefore choosing patients based on their sperm parameters for the DF test is advisable. We investigated the prevalence of sperm DNA damage in a group of infertile men with both normal and abnormal semen parameters in order to elucidate whether Sperm Chromatin Dispersion (SCD) analysis can add to the information obtained by routine semen analysis in explaining the causes of infertility.

\section{Materials and Methods}

In this study 152 patients, 64 patients in the working group and 88 patients in the control group were analyzed for analysis. The ejaculate samples were taken in accordance with the patient to whom the reason for the analysis of the ejaculate sample was previously explained. All patients have been in the Dukagjini Region in the Republic of Kosovo. The samples were collected from 2016/18. Sperm Chromatin Dispersion (SCD) analysis in the ejaculate was performed at the Biolab Zafi, Laboratory in Peja, in the Republic of Kosovo.

\subsection{Sperm Analysis}

Semen analysis was performed according to World Health Organization guidelines (WHO, 2010). Specimens were collected with assistance of the female partner after 3-5 days of sexual abstinence and analysis were performed after liquefaction, by using a light microscope. Sperm concentrations, morphology and motility were assessed before and after semen preparation. Sperm morphology was assessed according to Kruger's strict criteria after Papanicolaou staining. Sperm concentration was assessed by a Makler counting chamber (Bruckberg, Germany). Sperm motility was classified as either grades A, B or C and at least 100 spermatozoa were scored with a 40x objective. Total motility was calculated as the total of A and B motility rates.

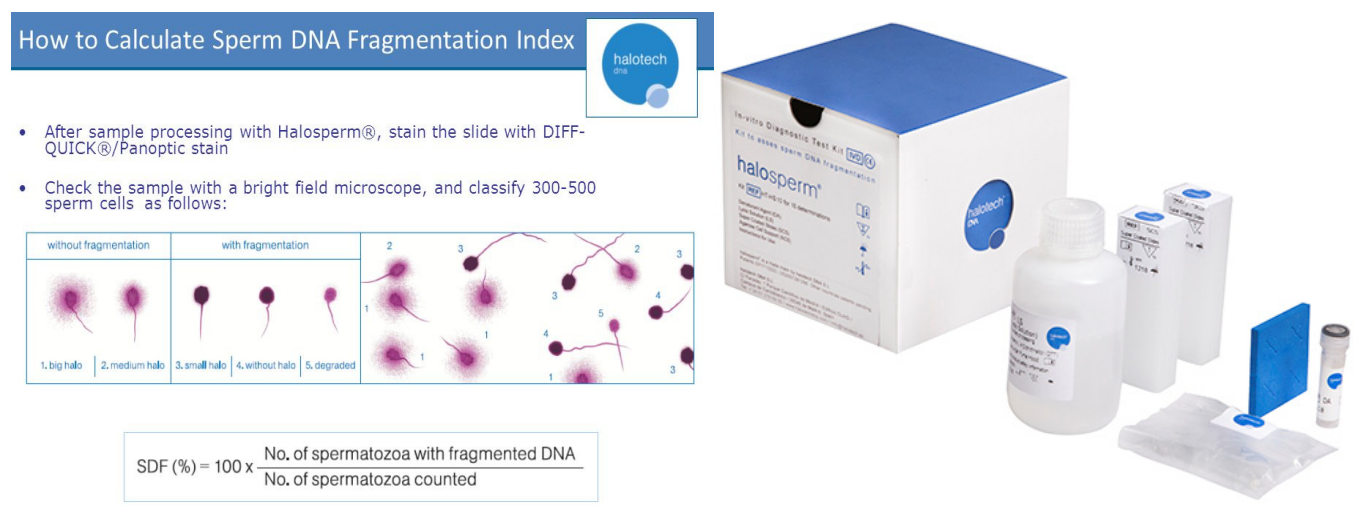

Figure 1. Halosperm ${ }^{\circledR}$ Halotech DNA

\subsection{Assessment of Sperm DNA Fragmentation (DF)}

Sperm Chromatin Dispersion (SCD) test, is an in vitro diagnostic kit that allows the measurement of DNA fragmentation in a fast, easy, and reproducible manner, without the need for complex laboratory equipment. Sperm Chromatin Dispersion (SCD) test allows for an improved evaluation of sperm quality, complementing the information offered by the conventional sperm analysis. Conventional semen analysis only studies spermatozoa concentration, motility, and morphology. It is an incomplete study, as it omits analysis of one of the most important parameters, the integrity of the DNA molecule. $15 \%$ of men considered to be infertile present normal sperm analyses, which makes a deeper assessment of the sample important. Sperm Chromatin Dispersion (SCD) test facilitates decision-making for medical professionals regarding which assisted reproduction technique is most appropriate for each couple. Sperm Chromatin Dispersion (SCD) test, based on a controlled DNA denaturation 
process to facilitate the subsequent removal of the proteins contained in each spermatozoon. In this way, normal spermatozoa create halos formed by loops of DNA at the head of the sperm, which are not present in those with damaged DNA. The images of halos generated with Sperm Chromatin Dispersion (SCD) are highly contrasted and can be evaluated precisely using conventional or fluorescent microscopy. The tails of the spermatozoa are preserved, so it is possible to discriminate them from other cell types that might be present in the ejaculate, such as leukocytes. In addition, Sperm Chromatin Dispersion (SCD) allows the visualization of spermatozoa that contain highly degraded DNA compared to other types of less aggressive damage.

\subsection{Statistical Analysis}

Data are reported as mean $\pm \mathrm{SD}$. The comparisons between groups were tested by student's t-test, ANOVA. A p-value less than $0.05 \%$ was considered statistically significant.

\section{Results}

Table 1. Sperm parameters and DNA fragmentation values in the two groups of subjects defined by the presence or not of abnormal standard semen parameters

\begin{tabular}{lcccc}
\hline & $\begin{array}{c}\text { Infertility groups (64 patients) } \\
\text { Average/Std }\end{array}$ & $\begin{array}{c}\text { Fertile groups (88 patients) } \\
\text { Average/Std }\end{array}$ & t-test & $\begin{array}{c}\text { Significant } \\
\mathrm{p}<0.05\end{array}$ \\
\hline Number in 1 million sperm & $19.67 \pm 19.79$ & $61.43 \pm 34.47$ & -8.247 & $\mathrm{p}<.00001$ \\
The general moving & $28.29 \pm 18.37$ & $57.15 \pm 10.83$ & -10.151 & $\mathrm{p}<.00001$ \\
Movement A & $14.09 \pm 10.86$ & $25.21 \pm 7.46$ & -7.359 & $\mathrm{p}<.00001$ \\
Movement B+C & $14.03 \pm 10.88$ & $31.73 \pm 10.09$ & -9.928 & $\mathrm{p}<.00001$ \\
Without moving & $71.59 \pm 18.34$ & $42.7 \pm 10.9$ & 11.554 & $\mathrm{p}<.00001$ \\
Normal morphology & $16.90 \pm 14.47$ & $42.6 \pm 15.2$ & -9.696 & $\mathrm{p}<.00001$ \\
Abnormal morphology & $83.09 \pm 14.47$ & $57.2 \pm 16.2$ & 8.991 & $\mathrm{p}<.00001$ \\
DNA- fragmentation (SDF\%) & $23.94 \pm 4.68$ & $15.13 \pm 4.02$ & 11.476 & $\mathrm{p}<.00001$ \\
\hline
\end{tabular}
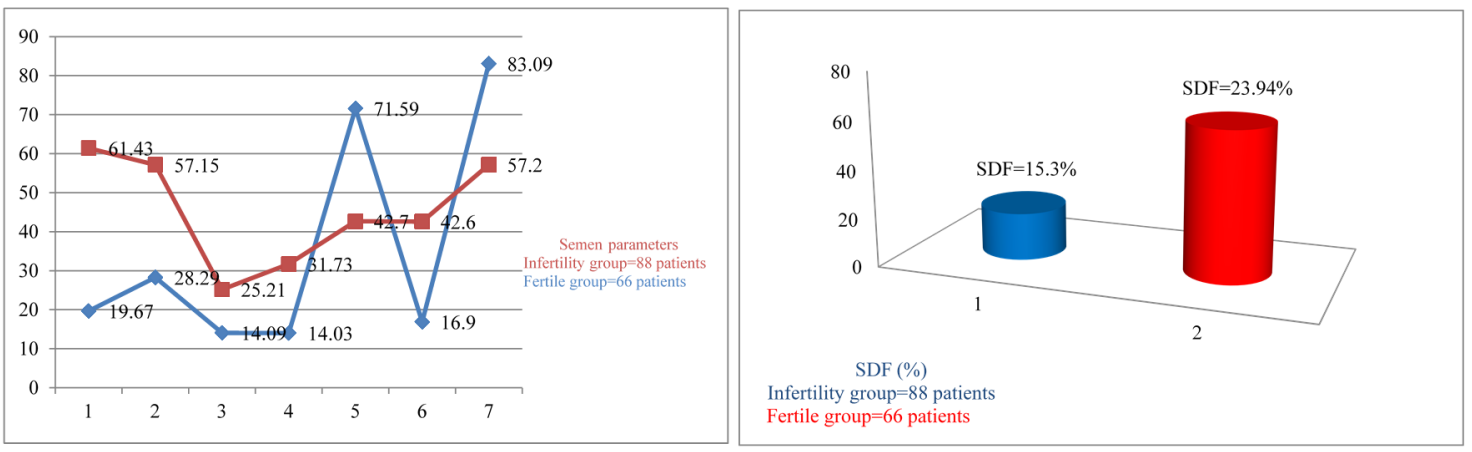

Figure 2. Graphical presentation of sperm parameters between two groups of patients taken for analysis

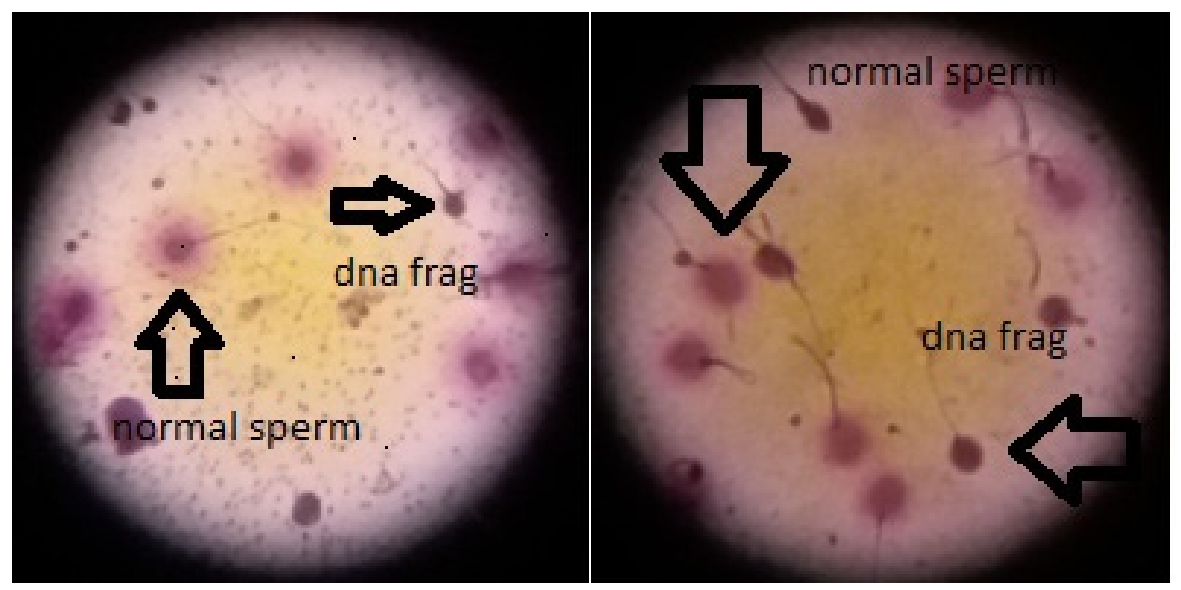

Figure 3. Photo by microscope during DNA fragmentation analysis in sperm cells 
From our research studies, we have achieved significant scores among the working group and control group across all sperm parameters, and DNA fragmentation. As shown in Table 1, there are significant differences in the mean of sperm concentration and sperm motility of fertile males were significantly higher than that of infertile males. The means of sperm concentration in fertile males was significantly higher than infertile males. Total sperm motility of fertile males also was significantly higher compared to infertile. Normal sperm morphology of fertile males was also significantly higher compared to infertile.

\section{Discussion}

\subsection{Assessment of Sperm DNA Fragmentation (DF)}

Sperm Chromatin Dispersion (SCD) test, is an in vitro diagnostic kit that allows the measurement of DNA fragmentation in a fast, easy, and reproducible manner, without the need for complex laboratory equipment. Sperm Chromatin Dispersion (SCD) test allows for an improved evaluation of sperm quality, complementing the information offered by the conventional sperm analysis. Conventional semen analysis only studies spermatozoa concentration, motility, and morphology. It is an incomplete study, as it omits analysis of one of the most important parameters, the integrity of the DNA molecule. This study indicate that sperm DNA fragmentation in infertile males are significantly higher than fertile males and sperms with abnormal morphology and low levels of motility have more abnormal DNA damages than motile and normal sperms. Our results have provided evidence of an important relationship between some of the semen parameters and sperm DNA fragmentation. Sperm DNA fragmentation as assessed by Sperm Chromatin Dispersion (SCD) demonstrated a very clear negative relationship between sperm motility and morphology. Control group had a lower percentage of sperm with DNA fragmentation compared with the infertile group (Irvine et al., 2000). Evaluated the association between semen parameters and DNA integrity among a group of infertile patients and a group of normozoospermic donors. They showed that semen parameters, especially sperm concentration, were inversely correlated with the comet assay parameters. (Sun et al., 1997) have demonstrated a significant negative correlation between semen parameters and DNA damage in sperm. Although several studies have shown the prognostic and diagnostic limitations of the routine semen parameters for the infertile couple, male infertility diagnosis is still based on the traditional semen analysis in routine clinical practice (Simon et al., 2010; Esteves et al., 2011). These results are consistent with the study of (Sergerie, et al., in 2005) that found mean value of sperm DFI in the infertile group was significantly higher than in the fertile group $(40.9 \pm 14.3 \%$ compared to $13.1 \pm 7.3 \%$ ) and the mean sperm concentration in infertile group also significantly lower compared to the fertile group $(62.9 \pm 33.2 \times 106 / \mathrm{ml}$ compared to $102.4 \pm 66.4 \times 106 / \mathrm{ml})$. Sperm DFI was significantly higher in patients with infertility compared to those of control $(22.2 \pm 5.6 \%$ vs. $16.7 \pm 0.7 \%$; $<0.05)$. From these results, it may be concluded that sperm DFI determination could be used to distinguish infertile men from fertile men (Diallo et al., 2015). Thus, it may be concluded that a man with sperm DFI of $\geq 26.1 \%$ has 2.84 times greater risk for infertility than men with sperm DFI of $<26.1 \%$. These results are consistent with a cohort study that found the most predictive cut-off point for pregnancy was sperm DFI of $>25.5 \%$ with a negative predictive value of $72.7 \%$ and the odds ratio for sperm DFI $<25.5 \%$ was 3.6 (95\% CI; 1.66, 7.82) (Lopez et al., 2013).

\section{Conclusion}

In summary, we have demonstrated that there was a negative correlation between DNA fragmentation, sperm motility, and morphology in infertile males. Also, there was a negative correlation between sperm motility and DNA fragmentation in infertile males. According to these results, we can conclude that testing sperm DNA fragmentation is helpful in selection of spermatozoa with the least amount of damage for use in assisted conception. In turn, this may alleviate the financial social and emotional problems associated with failed ART attempts. We conclude that sperm DNA fragmentation appears to be a useful technique to predict outcome in couples undergoing IVF/ICSI. Further studies are needed to confirm these encouraging preliminary findings as well to clarify the predictive potential of high-magnification sperm morphology for diagnostic purposes. To evaluate whether DFI $23.94 \pm 4.68 \%$ can be used to determine male infertility in our country by Sperm Chromatin Dispersion (SCD), it is necessary to carry out further large-scale research by other authors.

\section{Competing financial interests}

All authors declare no competing financial interests.

\section{References}

Agarwal, A., \& Said, T. M. (2003). Role of sperm chromatin abnormalities and DNA damage in male infertility. Human reproduction update, 9(4), 331-345.

Bungum, M., Humaidan, P., Axmon, A., Spano, M., Bungum, L., ... Giwercman, A. (2006). Sperm DNA integrity assessment in prediction of assisted reproduction technology outcome. Human reproduction, 22(1), 174-179. 
Diallo, M. S., Faye, O., Diallo, A. S., Diallo, Y., \& Diao, B. (2015). Increased DNA fragmentation in patients with infertility in Dakar (Senegal). Advances in Reproductive Sciences, 3(04), 97-105.

Esteves, S. C., \& Agarwal, A. (2011). Novel concepts in male infertility. International braz j urol, 37(1), 5-15.

Fernández, J. L., Muriel, L., Goyanes, V., Segrelles, E., Gosálvez, J., Enciso, M., ... \& De Jonge, C. (2005). Simple determination of human sperm DNA fragmentation with an improved sperm chromatin dispersion test. Fertility and sterility, 84(4), 833-842.

Gandini, L., Lombardo, F., Paoli, D., Caruso, F., Eleuteri, P., Leter, G., ... \& Spano, M. (2004). Full-term pregnancies achieved with ICSI despite high levels of sperm chromatin damage. Human Reproduction, 19(6), 1409-1417.

Høst, E., Ernst, E., Lindenberg, S., \& Smidt-Jensen, S. (2001). Morphology of spermatozoa used in IVF and ICSI from oligozoospermic men. Reproductive biomedicine online, 3(3), 212-215.

Irvine, D. S., Twigg, J. P., Gordon, E. L., Fulton, N., Milne, P. A., \& Aitken, R. (2000). DNA integrity in human spermatozoa: relationships with semen quality. Journal of andrology, 21(1), 33-44.

Kim, J. M., Ghosh, S. R., Weil, A. C., \& Zirkin, B. R. (2001). Caspase-3 and caspase-activated deoxyribonuclease are associated with testicular germ cell apoptosis resulting from reduced intratesticular testosterone. Endocrinology, 142(9), 3809-3816.

Li, T. C. (1998). Recurrent miscarriage: principles of management. Human reproduction (Oxford, England), 13(2), 478-482.

López, G., Lafuente, R., Checa, M. A., Carreras, R., \& Brassesco, M. (2013). Diagnostic value of sperm DNA fragmentation and sperm high-magnification for predicting outcome of assisted reproduction treatment. Asian journal of andrology, 15(6), 790-794.

Manicardi, G. C., Tombacco, A., Bizzaro, D., Bianchi, U., Bianchi, P. G., \& Sakkas, D. (1998). DNA strand breaks in ejaculated human spermatozoa: comparison of susceptibility to the nick translation and terminal transferase assays. The Histochemical Journal, 30(1), 33-39.

Marchettini, P., Solaro, C., Sommer, C., \& Cruccu, G. (2007). To become a pain specialist one has to understand the nervous system, yet the specialists of the nervous system still have a long way to go before understanding pain. Neurological Sciences, 28(4), 161-162.

Saleh, R. A., Agarwal, A., Nada, E. A., El-Tonsy, M. H., Sharma, R. K., Meyer, A., ... \& Thomas, A. J. (2003). Negative effects of increased sperm DNA damage in relation to seminal oxidative stress in men with idiopathic and male factor infertility. Fertility and Sterility, 79, 1597-1605.

Sergerie, M., Laforest, G., Bujan, L., Bissonnette, F., \& Bleau, G. (2005). Sperm DNA fragmentation: threshold value in male fertility. Human reproduction, 20(12), 3446-3451.

Simon, L., Brunborg, G., Stevenson, M., Lutton, D., McManus, J., \& Lewis, S. E. (2010). Clinical significance of sperm DNA damage in assisted reproduction outcome. Human reproduction, 25(7), 1594-1608.

Sun, J. G., Jurisicova, A., \& Casper, R. F. (1997). Detection of deoxyribonucleic acid fragmentation in human sperm: correlation with fertilization in vitro. Biology of reproduction, 56(3), 602-607.

Virro, M. R., Larson-Cook, K. L., \& Evenson, D. P. (2004). Sperm chromatin structure assay (SCSA $®$ ) parameters are related to fertilization, blastocyst development, and ongoing pregnancy in in vitro fertilization and intracytoplasmic sperm injection cycles. Fertility and sterility, 81(5), 1289-1295.

World Health Organization. (1999). Laboratory Manual for the Examination of Human Semen and SpermCervical Mucucs Interaction (4th ed.). Cambridge: Cambridge University Press.

Yılmaz, S., Zergeroğlu, A. D., Yılmaz, E., Sofuoglu, K., Delikara, N., \& Kutlu, P. (2010). Effects of sperm DNA fragmentation on semen parameters and ICSI outcome determined by an improved SCD test, Halosperm. Int J Fertil Steril, 4(2), 73-78.

\section{Copyrights}

Copyright for this article is retained by the author(s), with first publication rights granted to the journal.

This is an open-access article distributed under the terms and conditions of the Creative Commons Attribution license (http://creativecommons.org/licenses/by/4.0/). 